\title{
Presentazione dell'edizione italiana
}

Il continuo diffondersi di moderne apparecchiature e la sempre più frequente applicazione in ostetricia di sofisticate tecnologie affascina indubbiamente e giustamente le nuove leve di studenti e di neomedici che si accingono ad approfondire la nostra specialità. Ma l'ostetricia, per quella assoluta variabilità di evoluzione degli eventi clinici che la contraddistingue soprattutto in occasione del parto, non può prescindere da un buon bagaglio di esperienza pratica in termini di semeiotica ostetrica tradizionale.

L'aver approfondito al massimo gli aspetti tradizionali ma sempre attuali della nostra disciplina ritengo sia il pregio fondamentale del testo di Pschyrembel.

Nonostante tratti prevalentemente di ostetricia pratica in termini di semeiotica classica, questo libro non tralascia di riferire in modo succinto ma chiaro quanto le nuove tecniche applicate alla nostra specialità consentono.

Per tali motivi questo testo è particolarmente utile alle allieve ostetriche e agli studenti: per le allieve in virtù della calcolata ripetitività dei concetti espressi e al tempo stesso per lo schematismo quasi imperativo con cui essi vengono ribaditi; per lo studente in virtù della concomitante assoluta dovizia e chiarezza di informazioni, suggerimenti pratici, spiegazioni ed elementi diagnostici differenziali riportati.

Inoltre il testo, che non tralascia alcun tema fino al compimento del puerperio, è utile anche al medico di base che tuttora, anche se raramente, può trovarsi in situazioni ostetriche particolari che necessitano di tempestiva assistenza.

Giorgio D. Montanari 
